\title{
Comparison of behavioural and molecular effects of two different schizophrenia models induced by subchronic MK-801 administration in rats
}

\author{
Gökhan Ünal 1, Feyza Arıcioğlu 2,* \\ 1 Erciyes University, Faculty of Pharmacy, Department of Pharmacology, Kayseri, Turkey. \\ 2 Marmara University, Faculty of Pharmacy, Department of Pharmacology and Psychopharmacology Research Unit, \\ Istanbul, Turkey. \\ * Correspondence: feyza.aricioglu@gmail.com (F.A.); ORCID No: 0000-0003-4669-9382.
}

Received: 17 January 2018 / Revised: 15 February 2018 / Accepted: 25 February 2018

\begin{abstract}
Schizophrenia is a severe psychiatric disorder with about 1\% prevalance. NMDA receptor antagonists such as Phencyclidine (PCP) and MK-801 are commonly used for modeling schizophrenia in rodents. In literature, despite of the concensus about subchronic PCP administration (commonly 7 days, bi-daily administration followed by a 1 week washout period), there are different subchronic administration regimens for MK-801 beside 7 days, bidaily (MK-801-7), such as 14 days (MK-801-14) daily or 28 days daily injections. In this study, we aimed to compare two prevalant MK-801 models (MK-801-7 and MK-801-14, $0.2 \mathrm{mg} / \mathrm{kg}$ ) in both behavioural and molecular changes. Wistar Hannover rats grouped as control $(n=10)$, MK-801-14 $(n=8)$ and MK-801-7 $(n=8)$. Prepulse inhibition of acustic startle response (PPI), novel object recognition test (NORT), social interaction (SI) and Morris's water maze (MWM) tests were used for behavioural analyzes while real time polimerase chain reaction (Rt-PCR) was conducted for molecular analyzes of glutamic acid decarboxilase 67 (GAD67) and parvalbumin. Our results showed decreased PPI in MK-801-14 and MK801-7 groups. Moreover, in both models platform finding latencies were increased and swimming time in platform area was decreased in MWM. MK-801-14 and MK-801-7 reduced following and raised avoiding behaviours in SI. In Rt-PCR, GAD67 mRNA levels were decreased by MK-801-14 and MK-801-7 administrations. However, only MK-801-7 decreased discrimination index in NORT and parvalbumin mRNA levels. In this study, it has been showed that although MK-801-14 and MK-801-7 administrations revealed smiliar schizophrenia like symptoms in rats, MK-801-7 has partial superiories in certain aspects.
\end{abstract}

KEYWORDS: MK-801; GAD67; Parvalbumin; Schizophrenia

\section{INTRODUCTION}

Schizophrenia is one of the complex and devastating disease which is a common psychiatric disorder with approximately $1 \%$ prevalance [1]. Symptomology of schizophrenia are consisted of positive (hallucination and delusion), negative (asociality, amotivation, etc) and cognitive (learning, memory and attention deficits) symptoms. Even though neurobiology of schizophrenia could not be fully explained yet, it has been though that dopaminergic and glutamatergic dysfunctions are key factors for this pathology.

Glutamatergic hypofunction is one of the most accepted models to explain neurobiology of schizophrenia. According to this hypothesis, glutamatergic pathway betweeen the cortex and the brainstem controls gamma-aminobutyric acid (GABA)ergic, serotonergic and dopaminergic activities in healty subjects. It has been thought that glutamatergic hypoactivity (especially hypofunction of glutamatergic N-methyl-Daspartate (NMDA) receptors) might cause a decrease in GABAergic and increase in serotonergic activity in schizophrenic patients. As a result of these, dopaminergic activity increases in mesolimbic pathway, which is responsible for positive symptoms and decreases in mesocortical pathway, which is responsible for negative and cognitive symptoms [2]. This hypothesis originated from certain clinical observations that noncompetitive NMDA receptor antagonist Phencyclidine (PCP) reveals psychosis like behaviors in healthy volunteers [3]. These results are also confirmed by the observations that a non-competitive NMDA receptor antagonist and an anesthetic drug ketamine induce schizophrenia like behaviours in healty subjects [4].

How to cite this article: Ünal G, Arıcıoğlu F. Comparison of behavioural and molecular effects of two different schizophrenia models induced by subchronic MK-801 administration in rats. Marmara Pharm J. 2018; 22 (2): 199-208. 
In addition to this, evidence comes from genetic and post mortem studies show genetic abnormalities on NMDA receptor coding gene and decreased expression in related brain areas $[5,6]$.

GABAergic deficits are another core features of schizophrenia neurobiology. Postmortem studies have showed that gene expression of glutamic acid decarboxylase (GAD) enzyme, a major component of GABA synthesis, decreased in the brain of schizophrenic patients [7]. In addition to this, a number of studies indicated that parvalbumin containing GABAergic interneurons decreased in both hippocampus and prefrontal cortex $[8,9]$. These data showed that decreased GAD and parvalbumin expressions in brain are repeatable markers for schizophrenia. It has been thought that disrupted neuronal synchronization due to GABAergic deficits plays an important role in pathophysiology of schizophrenia.

Animal models can help for a better understanding of neurobiology of the disease and for developing new drugs. As pharmacological models of schizophrenia, NMDA receptor antagonists such as MK-801 are commonly used for modeling schizophrenia in rodents [10]. It has been demonstrated that acute administration of NMDA receptor antagonist mimics positive symptoms while subchronic administration reveals negative and cognitive symptoms of schizophrenia [11]. In literature, PCP is commonly administered bi-daily for 7 days with a week washout period while dose regimens of MK-801 varies in a wide range [12, 13]. Among these, daily injections for 14 days and bi-daily injections for 7 days are most frequent regimens for modeling schizophrenia in rodents. Therefore in this study we aimed to compare behavioural and molecular effects of two most chosen MK-801 models; daily administration for 14 days and bi-daily for 7 days in rats.

\section{RESULTS}

\subsection{Prepulse Inhibition of Acustic Startle Response (PPI)}

It has been found that MK-801-7 decreased PPI at the all prepulse intensities $(p<0.01$ for $+4 \mathrm{~dB}, \mathrm{p}<0.001$ for +8 and $+16 \mathrm{~dB})$ while MK-801-14 disrupted PPI at the $+8 \mathrm{~dB}(\mathrm{p}<0.001)$ and $+16 \mathrm{~dB}(\mathrm{p}<0.001)$ prepulse intensities compared with control (Figure 1a). In addition to this, both of MK-801-14 and MK-801-7 significantly decreased average PPI compared to control group $(\mathrm{p}<001)$ (Figure $1 b)$.
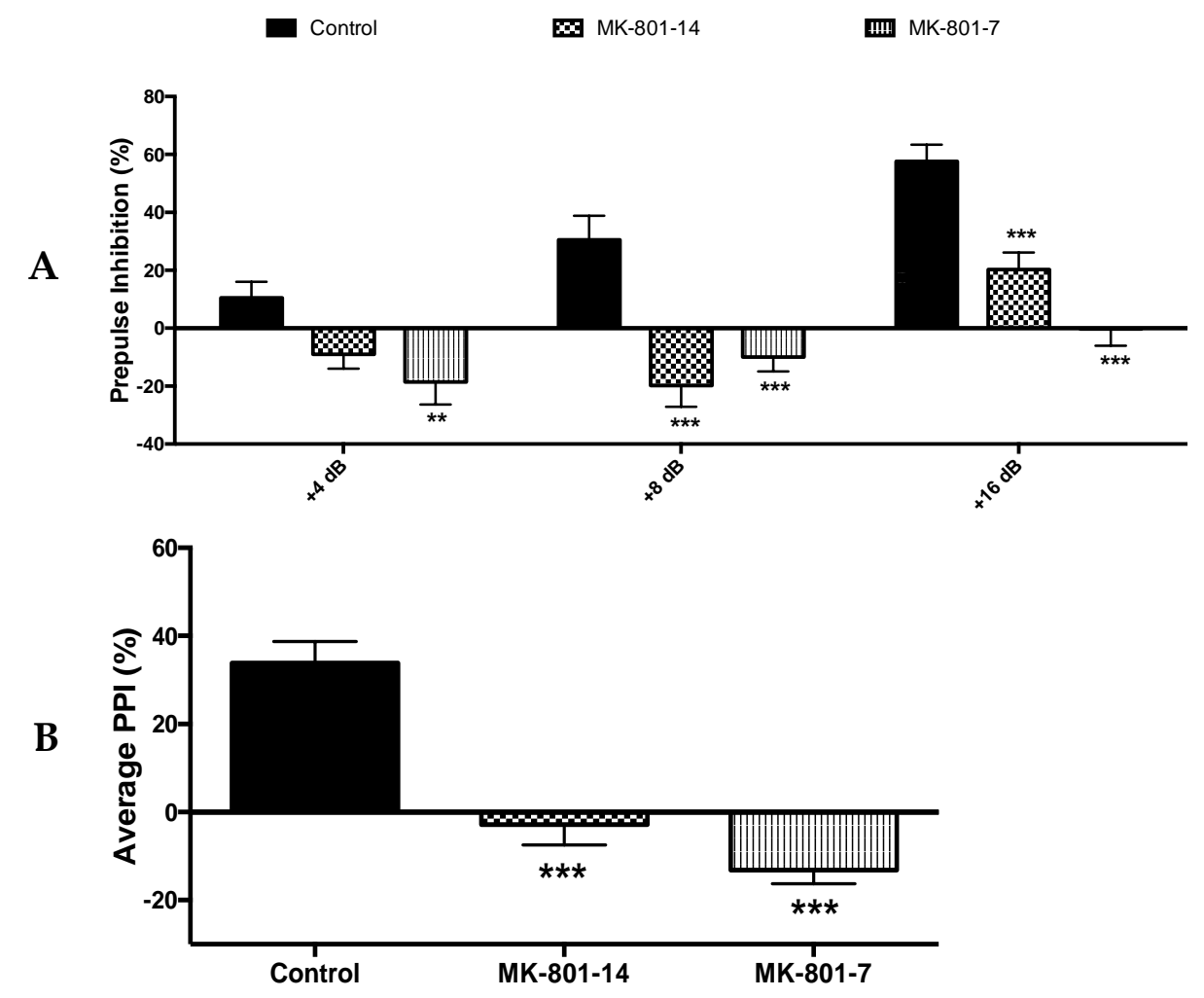

Figure 1a, b. The effects of different MK-801 models on PPI in different prepulse intensities (a) and average PPI (b). Data are expressed as mean \pm S.E.M, analyzed by using two-way (a) and one-way (b) ANOVA followed by Dunnett's post-hoc test. ${ }^{* *}: \mathrm{p}<0.01,{ }^{* * *}: \mathrm{p}<0.001$ compared with control group. 


\subsection{Novel Object Recognition Test (NORT)}

It has been found that time spent with novel object was higher than familiar one in all groups in NORT ( $p<0.001$ for control, $p<0.01$ for MK-801 groups)(Figure 2a). However, MK-801-7 had significantly lower discrimination index $(\mathrm{p}<0.01)$ compared to control group whereas the ratio was not statistically different than control in MK-801-14 group (Figure 2b).

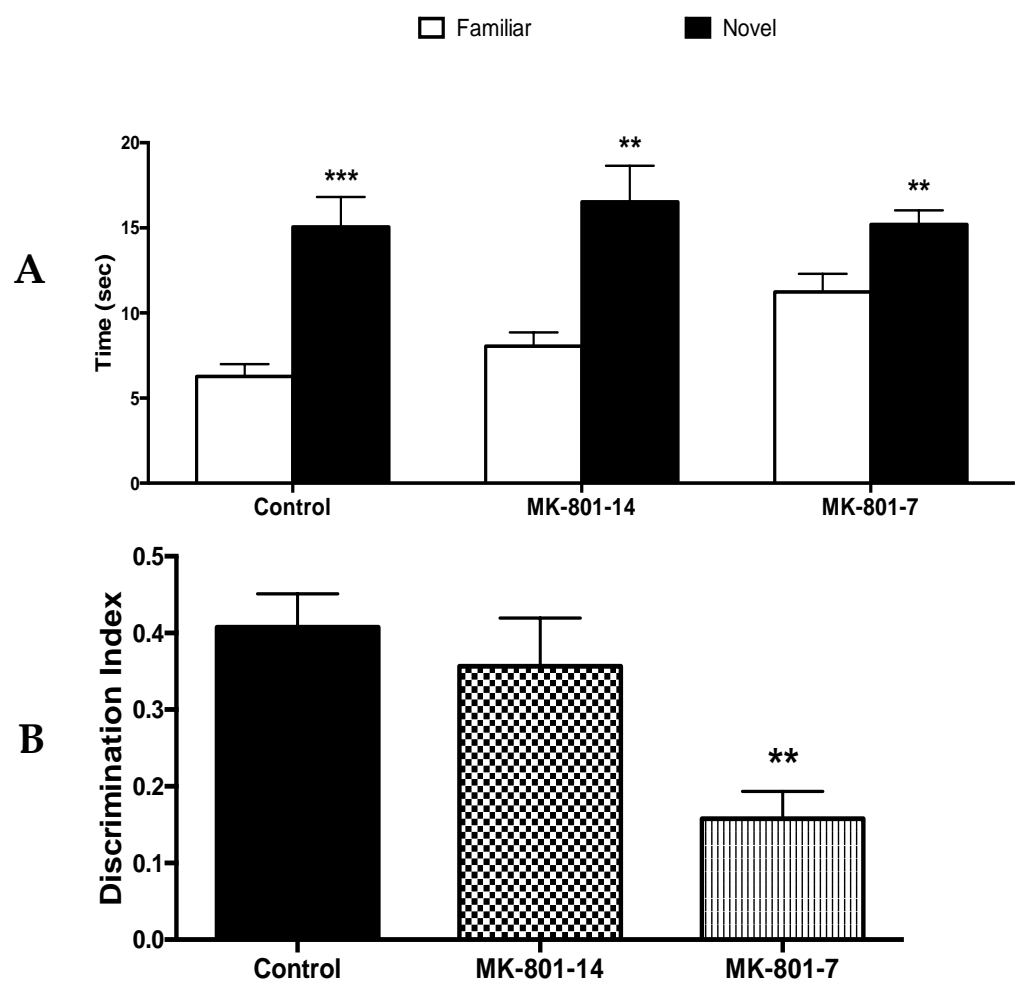

Figure 2a, b. Time spending with familiar and novel objects (a) and discrimination index of control, MK801-14 and MK-801-7 groups (b) in NORT. Data are expressed as mean \pm S.E.M, analyzed by paired Student's $t$ test (a) and one-way ANOVA followed by Dunnett's post-hoc test ${ }^{* *}$ :p $<0.01,{ }^{* * *}$ :p $<0.001$ compared with familiar object.

\subsection{Morris's Water Maze (MWM) Test}

In the acquisition period, MK-801-14 group's platform finding time was significantly higher than control in 1st $(\mathrm{p}<0.01)$, 2nd $(\mathrm{p}<0.001)$, 3rd $(\mathrm{p}<0.01)$ and 4th $(\mathrm{p}<0.001)$ days. MK-801-7 group found the platform later than control group in 1st $(p<0.01)$, 2nd $(p<0.001)$ and 4th $(p<0.01)$ days of MWM (Figure 3a). In the probe test, both of the MK-801-14 and MK-801-7 groups spent less time in the platform area compared with control group $(\mathrm{p}<0.01$ and $\mathrm{p}<0.05$, respectively)(Figure $3 \mathrm{~b})$.

\subsection{Social Interaction (SI) Test}

In SI test, no significant difference was found amoung groups in sniffing and climbing behaviours although there is certain decrease tendencies in MK-801 groups compared to control group (Figure 4a and Figure $4 \mathrm{~b}$ ). Both MK-801-14 and MK-801-7 groups markedly decreased following behavior compared to control group $(\mathrm{p}<0.05)$ (Figure 4c). In addition to these, both MK-801-14 and MK-801-7 groups had substantially increased avoiding behaviour compared to control group $(\mathrm{p}<0.001$ and $\mathrm{p}<0.05$, respectively)(Figure $4 \mathrm{~d}$ ).

\subsection{Molecular Analysis}

In Rt-PCR, MK-801-7 group had significantly lower hippocampal parvalbumin gene expression compared to control group whereas the expression level of MK-801-14 group was not statistically different than control group $(\mathrm{p}<0.01)$ (Figure 5a). In addition to this, GAD67 gene expressions markedly decreased in hippocampal tissues of MK-801-14 and MK-801-7 groups compared to control ( $p<0.05$ for each)(Figure 5b). 

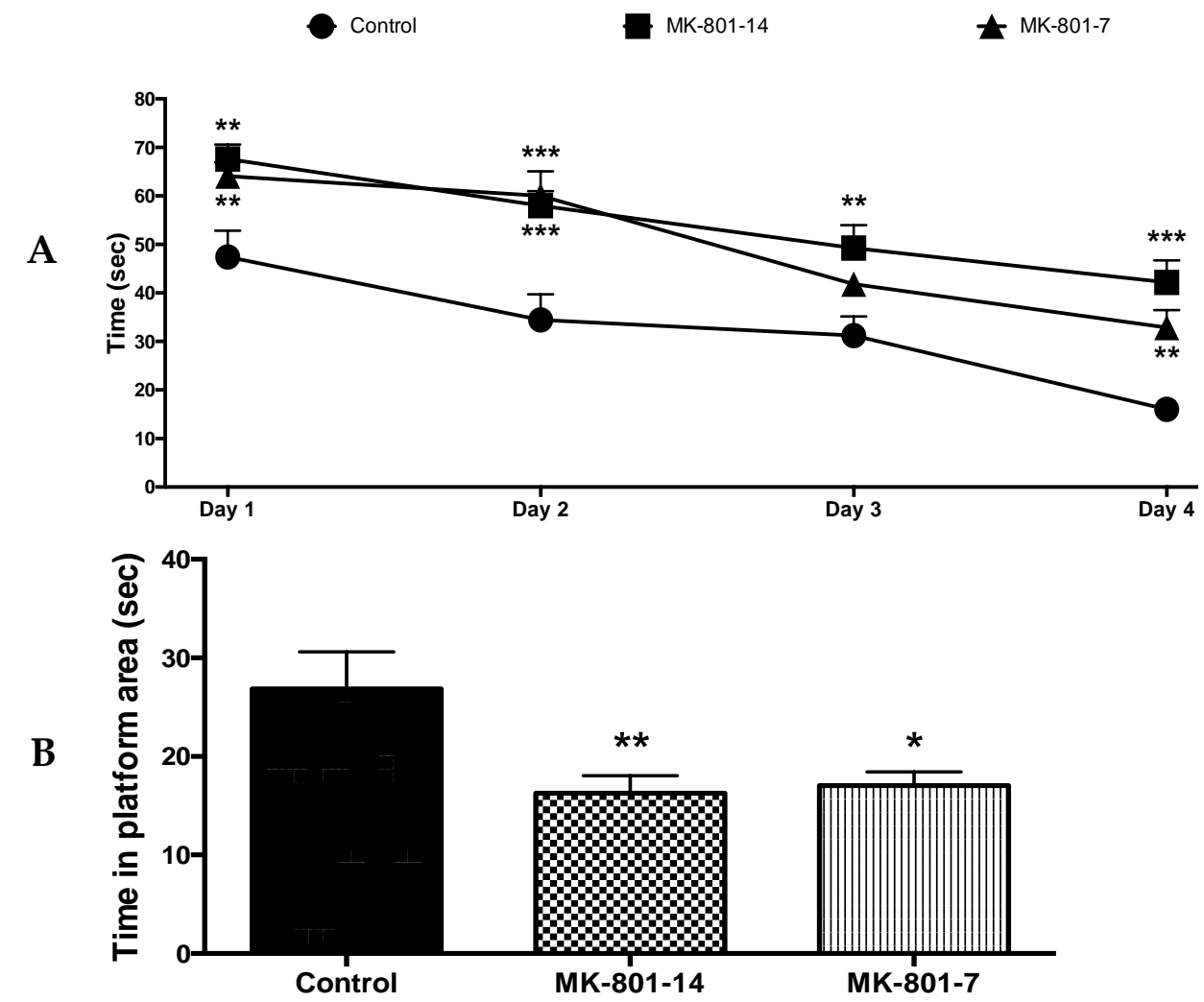

Figure 3a, b. Latency to platform finding (a) and the time spent in the platform area (b) of control, MK-80114 and MK-801-7 groups in MWM test. Data are expressed as mean, analyzed by using two-way (a) and one-way (b) ANOVA followed by Dunnett's post-hoc test. *:p<0.05, ${ }^{* *}$ :p $<0.01,{ }^{* * *}$ :p $<0.001$ compared with control group.

A

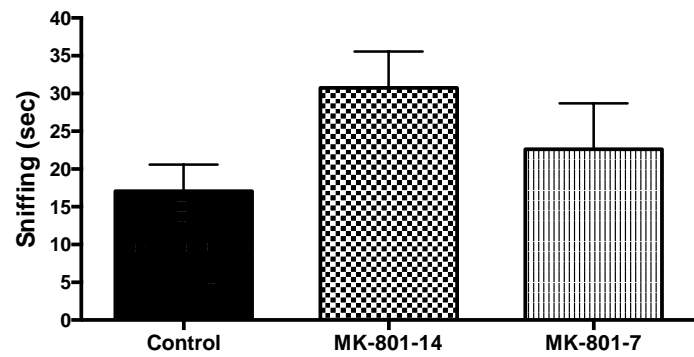

C

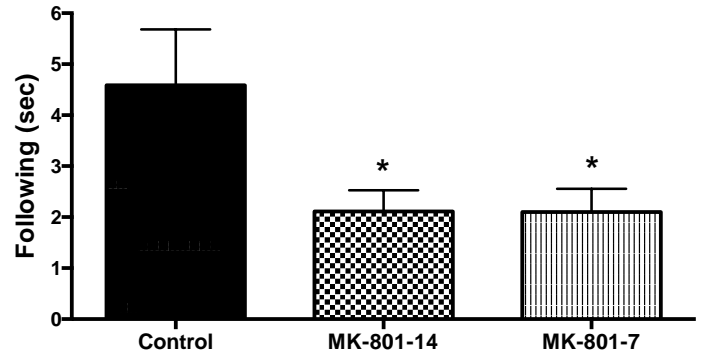

B

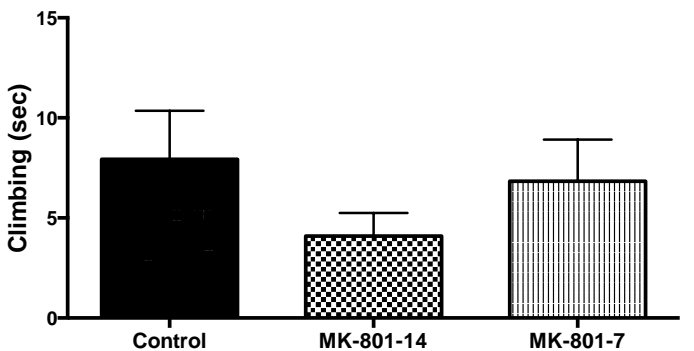

D

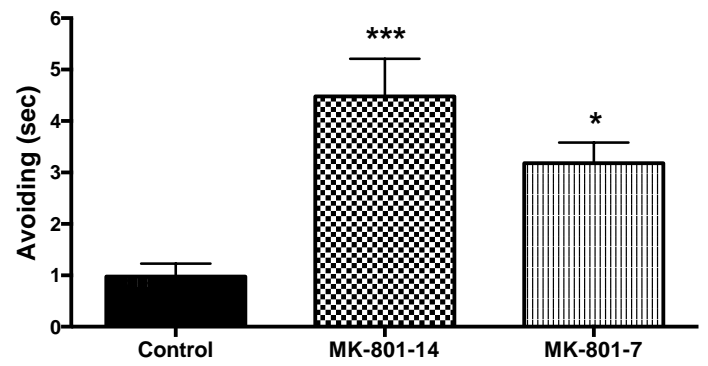

Figure 4a-d. Time spent for sniffing (a), climbing (b), following (c) and avoiding (d) behaviors of control, MK-801-14 and MK-801-7 groups groups in SI test. Data are expressed as mean \pm S.E.M and analyzed by using one-way ANOVA followed by Dunnett's post-hoc test. *:p<0.05 and ${ }^{* * *}: \mathrm{p}<0.001$ compared with control group. 
A

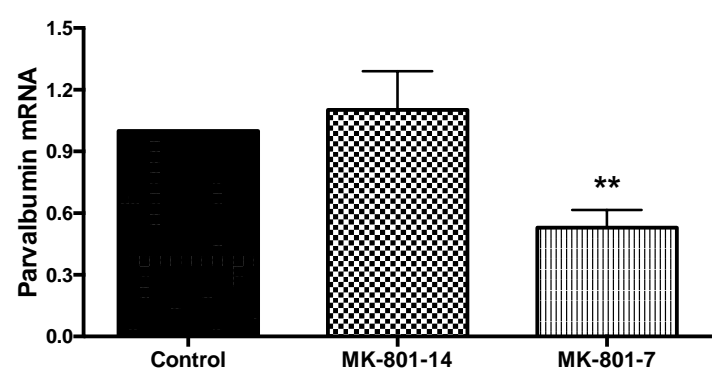

B

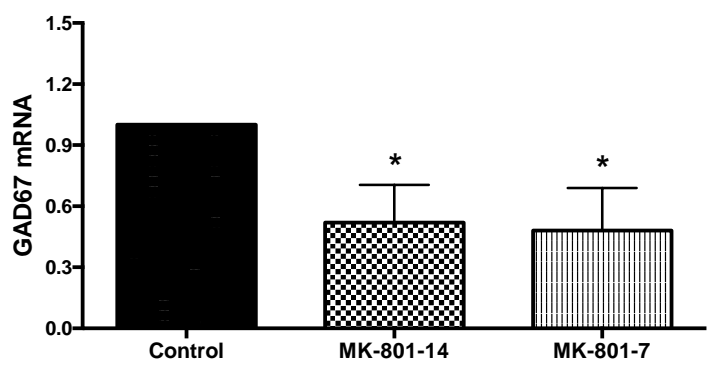

Figure 5a, b. Hippocampal parvalbumin (a) and GAD67 (b) mRNA levels of control, MK-801-14 and MK-801-7 groups. Data are expressed as mean \pm S.E.M and analyzed by using one-way ANOVA followed by Dunnett's post-hoc test. * $:$ p $<0.05$ and ${ }^{* *}$ : $p<0.01$ compared with control group.

\section{DISCUSSION}

Findings of the current study clearly showed that two different MK-801 regimens (daily 14 days and bidaily 7 days) revealed distinct behavioural and molecular effects in rats. 14 days administration of MK-801 disrupted sensorimotor gating, social functions and visiospatial learning and memory in behavioural tests. It also decreased GAD67 gene expression in hippocampus which was one of the repeatable data of schizophrenia. Bi-daily 7 days administration of MK-801 not only mimics the effects of MK-801-14 but also disrupted visual recognition memory and decreased parvalbumin gene expression in hippocampus of rats. It has well documented that PPI is a validated translational paradigm which shows sensorimotor gating in both human and rodents [14]. It has been thought that cortico-thalamo-pallido-striatal neuronal pathways play an important role for mediating PPI. Evidences have suggested that PPI was disrupted in certain neuropsychiatric diseases including schizophrenia and autism. In rodent studies, it has been shown that psycotomimetic drugs such as amphetamine and glutamatergic NMDA receptor antagonists such as MK-801 induce impairments of PPI [15]. It has been also proved that disruption of PPI by NMDA receptor antagonists depend on their acute effects and the effects are commonly transient $[15,16]$. In literature it has been seen that MK-801 impaired PPI at the doses $0.1-1 \mathrm{mg} / \mathrm{kg}$ in rats. The disruptive effect of MK- 801 on PPI has been confirmed by our previous studies in $0.2 \mathrm{mg} / \mathrm{kg}$ dose of MK-801 [17, 18]. In accordance with previous studies, our results showed that MK-801 administration disrupted sensorimotor gating in rats.

Cognitive dysfunction is one of the core deficits in schizophrenia. Measurement and Treatment Research to Imptove Cognition in Schizophrenia (MATRICS) initiative recommends NORT as a translational preclinical paradigm to research cognition in schizophrenia [19]. NORT bases on spontaneous exploratory behaviour of rodents and informs about visual episodic memory. It has been demonstrated that NORT performance is related to hippocampus and perirhinal cortex in rodents [19]. It has been indicated that subchronic administration of PCP generally impairs recognition memory in rodents after a week washout period whereas subchronic MK-801 administration has conflicting results for NORT [20]. It has been reported in previous studies that 14 days $(0.05,0.1$ and $0.2 \mathrm{mg} / \mathrm{kg})$ and 28 days $(0.25 \mathrm{mg} / \mathrm{kg})$ daily MK- 801 administrations did not impair recognition memory at NORT in rats $[12,18,20]$. Our results showed that 14 days daily administration did not affect NORT performance while 7 days bi-daily injections deteriorated recognition memory in rats. We have thought that the superior effect of bi-daily model might be related with more deterious effect of MK-801 on hippocampus in rats because our results showed decrease in hippocampal parvalbumin mRNA expressions in only MK-801-7 group while GAD67 mRNA expressions were found to be reduced in both groups. According to these results, we can hypothesize that especially parvalbumin containing GABAergic interneurons may play a crutial role for visual recognition memory and for this reason, bi-daily 7 days MK-801 injections were found to be superior than 14 days daily administration in this memory test.

It has been demonstrated that schizophrenic patients have severe visiospatial learning and memory deficits. MWM is commonly used to evaluate visiospatial learning and memory functions of rodents in behavioural studies. It has been thought that MWM reflects the revised form of Clinical Brief Visual Memory Test. It has been indicated that MWM performance is related to certain brain regions such as hippocampus, basal forebrain and cerebral cortex in rodents [21]. Studies suggested that NMDA receptor activity is neccessary for spatial memory and antagonists of this receptor reveal cognitive deficits in rodents [22-24]. In 
literature, it has been indicated that both of the 14 days, daily and 7 days, bi-daily injections of MK- 801 disrupted spatial learning and memory in MWM. In accordance with the previous studies, our results showed that both MK-801 groups deteriorated MWM performance of rats. We hypothesized that poor performance of the groups might be related with decreased GAD67 mRNA expressions beacuse it is well known that GABAergic interneurons play important role for mediating cognition in hippocampus.

Negative symptoms including social withdrawal are still unsolved problems for schizophrenic patients. It has been thought that frontal cortex and limbic regions play an important role for negative symptoms of schizophrenia [25]. Although modeling all of the negative symptoms is not easy, it has been approved that antagonism of NMDA receptor can reveal social deficits in rodents [20]. It was indicated that subchronic administration of NMDA receptor antagonists (especially PCP) decreased following, sniffing and climbing behaviours which represent socialization while its increased avoiding behaviour representing social withdrawal in rats [20, 26]. Effects of subchronic MK-801 administration on social interaction were investigated in small number of studies in rats. These studies demonstrated that both of the 7 days, bi-daily and 14 days, daily injections decreased social behaviours in rats [27, 28]. In this manner, our results showing the reduced sociality and increased social withdrawal in both group were parallel with previous studies.

There are certain limitations of this study. Both MK-801-14 and MK-801-7 models were shown to demonstrate behavioral deficits and neurobiological alterations relevance to schizophrenia, therefore both models were considered to well meet the face and construct validity criteria. However, considering the fact that an antpsychotic-treated group was not included in our experimental design, therefore predictive validity of this model was not shown in the present study. In this context, it is of note to mention that this issue was repeatedly addressed by previous studies conducted in subchronic NMDA receptor antagonist models showing that atypical antipsychotic treatments reversed schizophrenia like deficits in rats [11]. Secondly, our results showing that MK-801 administration downregulated parvalbumin and GAD67 mRNA levels requires further investigations by using protein expression analysis which would provide better interperation of our results regarding the significance of the MK- 801 model on the functional activity of these parameters.

\section{CONCLUSION}

In conclusion, MK-801 administration is one of the most commonly used model to mimic schizophrenia like behaviours in rats by a wide range of doses and administration regimens. Up to present there is no study showing the superiority of any MK-801 models in terms of inducing behavioural and molecular deficits. To our knowledge, this is the first study that comperatively investigates molecular and behavioural effects of the most commonly used subchronic MK-801 models of schizophrenia in rats. Our results demonstrated that 7 days bi-daliy administration had better profile on behavioural and molecular parameters than 14 days daily regimen therefore 7 days bi-dialy administrations of MK- 801 might be a better choice for modeling schizophrenia in rats.

\section{MATERIALS AND METHODS}

\subsection{Animals and Housing}

All experiments documented in this study were conducted in accordance with the Regulation of Animal Research Ethics Committee in Turkey (6 July 2006, Number 26220). This study was approved by Marmara University Animal Research Ethics Committee (4 January 2016, approval number: 017.2016.mar). Male Wistar Hannover rats (8-12 weeks and 180-250 g) were used in this study. The laboratory had optimum room conditions $\left(22^{\circ} \mathrm{C}\right.$ and $12 / 12$ light cycle). Animals fed ad libitum bait and water. Experiments were done at the light phase (09 am - 02 pm) of light/dark cycle. Experiments were performed after two weeks habituation period of rats to the laboratory and three days of handling process. Animals were divided into three groups; control (saline, $\mathrm{n}=10)$, MK-801-14 (0.2 mg/kg, 14 days, once a day, $\mathrm{n}=8)$ and MK-801-7 (0.2 mg/kg, 7 days, twice a day, $\mathrm{n}=8)$.

\subsection{Drugs}

MK-801 ((+)-MK-801 hydrogen maleate, Cayman) was dissolved in saline. Saline and MK-801 were intraperitoneally injected to rats at a volume of $0,1 \mathrm{ml} / 100 \mathrm{~g}$. MK-801 was injected subcutaneously only before the prepulse inhibition of acustic startle response (PPI) test. 


\subsection{Experimental Design and Treatments}

MK-801 $(0.2 \mathrm{mg} / \mathrm{kg})$ was injected once a day for 14 days or twice a day for 7 days. The dose of MK-801 $(0.2 \mathrm{mg} / \mathrm{kg})$ was selected according to our previous studies and it was used in both of MK-801-14 and MK801-7 groups. PPI test was performed 15 min after the last doses of MK-801. To avoid the acute effects of MK801, novel object recognition test (NORT), social interaction (SI) and Morris's water maze (MWM) tests have been performed after a seven day washout period. All rats were decapitated $24 \mathrm{~h}$ after the behavioral tests and hippocampus was dissected for molecular analyzes.

\subsection{Prepulse Inhibition of Acustic Startle Response (PPI)}

Acustic startle responses of rats were measured by SR-LAB system (San Diego Instruments, San Diego, CA). This system is consisted of a control unit, a computer with SR LAB software, two identical startle response chambers $(39 \times 38 \times 58 \mathrm{~cm})$ and plexiglass cylinder cages $(8.8 \mathrm{~cm}$ diameter, $25 \mathrm{~cm}$ lenght) and loudspeakers in the chambers.

The test was performed according to previous studies [15]. An habituation process was conducted before the testing day. On the habituation day, the rats were exposed to background noise $(70 \mathrm{~dB})$ for $5 \mathrm{~min}$ and then five startle stimulus to testing of hearing and startle responses. On the test day, the rats were firstly exposed to background noises for $5 \mathrm{~min}$. After this period, trials were applied in three blocks. Block 1 was consisted of five presentations of $120 \mathrm{~dB}(40 \mathrm{~ms})$ pulse-alone trials. In block 2, there were 50 pseudo-random trials (average intertrial intervals of $15 \mathrm{~s})$ consisting of ten presentations of each prepulse + pulse trials $(74,78$, $86 \mathrm{~dB}$ prepulses ( $20 \mathrm{~ms}$ for each) $100 \mathrm{~ms}$ before $120 \mathrm{~dB}$ pulse), ten presentations of $120 \mathrm{~dB}$ pulse-alone and ten presentations of no stimulus. In block 3, rats were exposed to five presentations of pulse-alone trials. For evaluating the percent inhibition of startle, only block 2 data was used. Prepulse inhibition of startle response was computed by the following formula: \%PPI= $100-(\mathrm{PP}+\mathrm{P}) /(\mathrm{P}) \times 100$. "PP+P" means the startle response after presentation of prepulse and pulse stimulus whereas " $\mathrm{P}$ " means startle response after pulse-alone stimulus. Average PPI was calculated as "Average PPI= (PPI74dB + PPI78dB + PPI86dB) / 3".

\subsection{Novel Object Recognition Test (NORT)}

NORT was conducted in a black plexiglass chamber $(50 \times 50 \times 30 \mathrm{~cm})$ at half-light room conditions. The chamber was cleaned with $70 \%$ ethanol after each animal. The experiment was performed in two days; habituation and test days. This experiment was performed according to previous studies [29] and was summerized as follows:

Habituation (Day 1): All animals in the same group were allowed to acclimate to the test apparatus for $60 \mathrm{~min}$. There was not any object in habituation period. The process was conducted per group.

Test (Day 2): The test was performed in two trials (3 min) with 1 hour interval. In trial 1 (Familiarisation, T1) rats was allowed to explore two identical objects (A for each) placed into opposite corners of chamber during $3 \mathrm{~min}$. In trial 2 (Retention, T2), one of the identical objects was changed with a novel one (B) and rats spent $3 \mathrm{~min}$ for exploration. The sizes and shapes of familiar and novel objects were different but comparible with each other (abouth $10 \mathrm{~cm}$ height and $4 \mathrm{~cm}$ diameter). Sniffing, licking and touching behaviours was assumed as exploratory behavior. All trials were recorded by a camera placed above the apparatus. Exploration time (E) was scorred by a blind researcher. Discrimintion index (DI) was calculated as the following formula: $\mathrm{DI}=(\mathrm{EB}-\mathrm{EA}) /(\mathrm{EB}+\mathrm{EA})$.

\subsection{Morris Water Maze (MWM) Test}

MWM consists of a cylinder pool $(160 \mathrm{~cm}$ diameter and $50 \mathrm{~cm}$ wall heigh), a platform $(12 \mathrm{~cm}$ diameter and $30 \mathrm{~cm}$ heigh) and four cues (different shape and colour cardboard). MWM was filled with water $\left(25 \pm 1^{\circ} \mathrm{C}\right)$ until $1 \mathrm{~cm}$ above of platform. Pool was theoretically divided into four quarters and it was presumed that each quarter represents different directions (North, South, West and East). Cues were posted the opposite of the each direction. Platform was placed in the middle of South-East quarter. Noldus Ethovision XT® was used for tracking the animals and recording the trials. The water was encoulered with Mixol ${ }^{\circledR}$ to black because of facilitating the software's tracking to animals.

In acquisition period, rats were trained for each direction per day during 4 days. In a trial, rats were reliased to water from selected direction and allowed $75 \mathrm{~s}$ for finding the platform. Experimenter placed the rats which could not find the platform in $75 \mathrm{~s}$ on the platform for $20 \mathrm{~s}$. The trials were performed from 4 different directions per rat in a day. The order of the directions was different from each other in every day. Platform finding latencies were measured in acquisition trials. On the probe test (5th day), the platform was 
removed and rats were reliased to maze from North. The rats were allowed to swim freely for $60 \mathrm{~s}$ and swimming time in the platform quarter (Notrh-East) was evaluated in the probe test [30]. All trials were recorded with a camera and both of the platform finding latencies and swimming pattern was calculated with Noldus Ethovision XT®.

\subsection{Social Interaction (SI) Test}

SI was performed in a black plexiglass chamber $(50 \times 50 \times 30 \mathrm{~cm})$ at the half-light room conditions. The plexiglass chambers was cleaned with $70 \%$ ethanol before each rat.

In this test, two unfamiliar rats which are in the same treatment group but from different cages were placed in the chamber for $10 \mathrm{~min}$. To discriminate a rat from other one, one of them was coloured with temporary ink before the test. Social behaviors of each rat were investigated seperately. All experiments were recorded by a camera and sniffing, climbing, following and avoiding behaviors were scored by a blind resercher [19].

\subsection{RNA Isolation and $q R T-P C R$}

Total RNA was isolated using RNAzol isolation reagent (MRC, Canada, RN190) from the hippocampal tissue according to the manufacturer's instructions. Total RNA $(1 \mu \mathrm{g})$ was reverse-transcribed to cDNA using a Transcriptor High Fidelity cDNA Synthesis Kit (Jena Bioscience, Germany). Real-time PCR was carried out using a Agilent Stratagene 3005P system (Agilent, USA). To quantify cDNA, qPCR was performed using FastStart Essential DNA probe master mix (Jena Bioscience, Germany) and catalogue assay kits, which consist the primers and probes for determination of GAD67, parvalbumin and GAPDH (Table 1). For each sample the level of target gene transcripts was normalized to GAPDH. Primer series of target genes in Rt-PCR

$\mathrm{Cp}$ values were calculated using the software after Rt-PCR and. $2^{\wedge}$-ddCT method was used for relative quantification.

$$
2^{\wedge} \text {-ddCT }=2-(C t \text { target }-\mathrm{Ct} \text { target reference) } / 2 \text { - (Ct control }- \text { Ct control reference }) \times 100
$$

Table 1. Primer series of target genes in Rt-PCR

\begin{tabular}{lll}
\hline Gene & Primer (Forward) & Primer (Reverse) \\
\hline PVALB & 5'd TGCGGATGATGTGAAGAAGGT 3' & 5' d CAGCCATCAGCGTCTTTGTTT 3' \\
GAD1 & 5' d GGAAGCATCGCCACAAACTC 3' & 5' d TGACCAGGATGGCAGAACAC 3' \\
GAPDH & 5' d GCCCCCGGTTTCTATAAATTG 3' & 5' d GTCGAACAGGAGGAGCAGAGA 3' \\
\hline
\end{tabular}

\subsection{Statistical Analysis}

All statistical analyses were performed by GraphPad Prism 6.0. Statistical analyses were performed by one-way analysis of variance (ANOVA) in NORT, SI, MWM and Rt-PCR analyses. In PPI and MWM acquisition trials, two-way ANOVA was conducted for statistical analyses. Dunnett's post hoc test was used for comparison of the different groups in all experiments. Paired Student's $t$ test was also used to compare exploratory behaviors of rats for the familiar and novel objects in NORT. Data were presented as mean \pm standart error of mean (S.E.M) for all experiments. Data were assumed statistical significant when $p$ value was lower than 0.05 .

Author contributions: Concept -G.Ü.; Design - G.Ü.; Supervision - F.A.; Resource - F.A.; Materials - G.Ü., F.A.; Data Collection and/or Processing -G.Ü.; Analysis and/or Interpretation - F.A.; Literature Search - G.Ü., F.A.; Writing -G.Ü., F.A.; Critical Reviews - G.Ü., F.A.

Conflict of interest statement: The authors declared no conflict of interest" in the manuscript.

\section{REFERENCES}

[1] Tandon R, Keshavan MS, Nasrallah HA. Schizophrenia, "just the facts" what we know in 2008. 2. Epidemiology and etiology. Schizophr Res. 2008; 102(1-3): 1-18.

[2] Schwartz TL, Sachdeva S, Stahl SM. Glutamate neurocircuitry: theoretical underpinnings in schizophrenia. Front Pharmacol. 2012; 3: 195.

[3] Snigdha S, Neill JC. Efficacy of antipsychotics to reverse phencyclidine-induced social interaction deficits in female rats--a preliminary investigation. Behav Brain Res. 2008; 187(2): 489-494. 
[4] Lahti AC, Weiler MA, Tamara M, Parwani A, Tamminga CA. Effects of ketamine in normal and schizophrenic volunteers. Neuropsychopharmacology. 2001; 25(4): 455-467.

[5] Akbarian S, Sucher N, Bradley D, Tafazzoli A, Trinh D, Hetrick WP, Potkin SG, Sandman CA, Bunney WE Jr, Jones EG. Selective alterations in gene expression for NMDA receptor subunits in prefrontal cortex of schizophrenics. J Neurosci. 1996; 16(1): 19-30.

[6] Kristiansen L, Beneyto M, Haroutunian V, Meador-Woodruff J. Changes in NMDA receptor subunits and interacting PSD proteins in dorsolateral prefrontal and anterior cingulate cortex indicate abnormal regional expression in schizophrenia. Mol Psychiatry. 2006; 11(8): 737-747.

[7] Lewis DA, Hashimoto T, Volk DW. Cortical inhibitory neurons and schizophrenia. Nat Rev Neurosci. 2005; 6(4): 312324.

[8] Lewis DA, Curley AA, Glausier JR, Volk DW. Cortical parvalbumin interneurons and cognitive dysfunction in schizophrenia. Trends Neurosci. 2012; 35(1): 57-67.

[9] Heckers S, Konradi C. GABAergic mechanisms of hippocampal hyperactivity in schizophrenia. Schizophr Res. 2015; 167(1): 4-11.

[10] Jones CA, Watson DJ, Fone KC. Animal models of schizophrenia. Br J Pharmacol. 2011; 164(4): 1162-1194.

[11] Neill JC, Harte MK, Haddad PM, Lydall ES, Dwyer DM. Acute and chronic effects of NMDA receptor antagonists in rodents, relevance to negative symptoms of schizophrenia: a translational link to humans. Eur Neuropsychopharmacol. 2014; 24(5): 822-835.

[12] Li JT, Su YA, Guo CM, Feng Y, Yang Y, Huang RH, Si TM. Persisting cognitive deficits induced by low-dose, subchronic treatment with MK-801 in adolescent rats. Eur J Pharmacol. 2011; 652(1): 65-72.

[13] Beninger RJ, Forsyth JK, Van Adel M, Reynolds JN, Boegman RJ, Jhamandas K. Subchronic MK-801 behavioural deficits in rats: partial reversal by the novel nitrate GT 1061. Pharmacol Biochem Behav. 2009; 91(4): 495-502.

[14] Callahan PM, Terry Jr AV, Tehim A. Effects of the nicotinic $\alpha 7$ receptor partial agonist GTS-21 on NMDAglutamatergic receptor related deficits in sensorimotor gating and recognition memory in rats. Psychopharmacology (Berl). 2014; 231(18): 3695-3706.

[15] Mansbach RS, Geyer MA. Effects of phencyclidine and phencyclidine biologs on sensorimotor gating in the rat. Neuropsychopharmacology. 1989; 2(4): 299-308.

[16] Mansbach RS, Geyer MA, Braff DL. Dopaminergic stimulation disrupts sensorimotor gating in the rat. Psychopharmacology (Berl). 1988; 94(4): 507-514.

[17] Unal G, Aricioglu F. A-582941, cholinergic alpha 7 nicotinic receptor agonist, improved cognitive and negative symptoms of the sub-chronic MK-801 model of schizophrenia in rats. Psychiatry and Clinical Psychopharmacology. 2018; 28(1): 4-13.

[18] Unal G, Ates A, Aricioglu F. Agmatine attenuated cognitive and social deficits in subchronic MK-801 model of schizophrenia in rats. Psychiatry and Clinical Psychopharmacology. $25 \quad$ Jan 2018. http://dx.doi.org/10.1080/24750573.2018.1426696.

[19] Neill JC, Barnes S, Cook S, Grayson B, Idris NF, McLean SL, Snigdha S, Rajagopal L, Harte MK. Animal models of cognitive dysfunction and negative symptoms of schizophrenia: focus on NMDA receptor antagonism. Pharmacol Ther. 2010; 128(3): 419-432.

[20] Li JT, Feng Y, Su YA, Wang XD, Si TM. Enhanced interaction among ErbB4, PSD-95 and NMDAR by chronic MK-801 treatment is associated with behavioral abnormalities. Pharmacol Biochem Behav. 2013; 108: 44-53.

[21] D'Hooge R, De Deyn PP. Applications of the Morris water maze in the study of learning and memory. Brain Res Rev. 2001; 36(1): 60-90.

[22] Janhunen S, Svärd H, Talpos J, Kumar G, Steckler T, Plath N, Lerdrup L, Ruby T, Haman M, Wyler R, Ballard TM. The subchronic phencyclidine rat model: relevance for the assessment of novel therapeutics for cognitive impairment associated with schizophrenia. Psychopharmacology (Berl). 2015; (21-22): 4059-4083.

[23] Didriksen M, Skarsfeldt T, Arnt J. Reversal of PCP-induced learning and memory deficits in the Morris' water maze by sertindole and other antipsychotics. Psychopharmacology (Berl). 2007; 193(2): 225-233.

[24] Wass C, Archer T, Pålsson E, Fejgin K, Klamer D, Engel JA, Svensson L. Effects of phencyclidine on spatial learning and memory: nitric oxide-dependent mechanisms. Behav Brain Res. 2006; 171(1): 147-153. 
[25] Semkovska M, Bedard M, Stip E. Hypofrontality and negative symptoms in schizophrenia: synthesis of anatomic and neuropsychological knowledge and ecological perspectives. L'encephale. 2001; 27(5): 405-415.

[26] Kamińska K, Rogóż Z. The effect of combined treatment with risperidone and antidepressants on the MK-801induced deficits in the social interaction test in rats. Pharmacol Rep. 2015; 67(6): 1183-1187.

[27] Seillier A, Giuffrida A. Evaluation of NMDA receptor models of schizophrenia: divergences in the behavioral effects of sub-chronic PCP and MK-801. Behav Brain Res. 2009; 204(2): 410-415.

[28] Matsuoka T, Sumiyoshi T, Tanaka K, Tsunoda M, Uehara T, Itoh H, Kurachi M. NC-1900, an arginine-vasopressin analogue, ameliorates social behavior deficits and hyperlocomotion in MK-801-treated rats: Therapeutic implications for schizophrenia. Brain Res. 2005; 1053(1): 131-136.

[29] Bevins RA, Besheer J. Object recognition in rats and mice: a one-trial non-matching-to-sample learning task to study 'recognition memory'. Nat Protoc. 2006; 1(3): 1306-1311.

[30] Morris R. Developments of a water-maze procedure for studying spatial learning in the rat. J Neurosci Methods. 1984; 11(1): 47-60.

This is an open access article which is publicly available on our journal's website under Institutional Repository at http://dspace.marmara.edu.tr. 